\title{
Oxidation resistance 1 functions in the maintenance of cellular survival and genome stability in response to oxidative stress-independent DNA damage
}

\author{
Ako Matsui ${ }^{1,2}$, Kazunari Hashiguchi ${ }^{1,3}$, Masao Suzuki $^{4}$ and Qiu-Mei Zhang-Akiyama ${ }^{1 *}$
}

\begin{abstract}
Background: DNA damage is generated by various intrinsic and extrinsic sources such as reactive oxygen species (ROS) and environmental mutagens, and causes genomic alterations. DNA damage response (DDR) is activated to induce cell cycle arrest and DNA repair. Oxidation resistance 1 (OXR1) is a protein that defends cells against oxidative stress. We previously reported that OXR1 protein functions in the regulation of G2-phase cell cycle arrest in cells irradiated with gamma-rays, suggesting that OXR1 directly responds to DNA damage.

Purpose: To clarify the functions of OXR1 against ROS-independent DNA damage, HeLa and OXR1-depleted HeLa cells were treated with heavy-ion beams and the ROS-independent DNA-damaging agent methyl methanesulfonate (MMS).

Results: First, OXR1-depleted cells exhibited higher sensitivity to MMS and heavy-ion beams than control cells. Next, OXR1 depletion increased micronucleus formation and shortened the duration of G2-phase arrest after treatment with MMS or heavy-ion beams. These results suggest that OXR1 functions in the maintenance of cell survival and genome stability in response to DNA damage. Furthermore, the OXR1 protein level was increased by MMS and heavy-ion beams in HeLa cells.

Conclusions: Together with our previous study, the present study suggests that OXR1 plays an important role in the response to DNA damage, not only when DNA damage is generated by ROS.
\end{abstract}

Keywords: OXR1, DNA damage response, Cell cycle checkpoint, Cellular survival, Protein expression

\section{Introduction}

DNA damage is generated by various intrinsic and extrinsic sources such as reactive oxygen species (ROS) and environmental mutagens, and can cause genomic alterations, leading to cancer and neuronal diseases. Oxidative stress is the condition in which ROS are

\footnotetext{
* Correspondence: qmzhang@kingyo.zool.kyoto-u.ac.jp

'Laboratory of Stress Response Biology, Department of Zoology, Division of Biological Sciences, Graduate School of Science, Kyoto University, Kitashirakawa Oiwake-cho, Sakyo-ku, Kyoto 606-8502, Japan

Full list of author information is available at the end of the article
}

accumulated excessively $[1,2]$. In most eukaryotes, Oxidation resistance 1 (OXR1) protein protects cells and organisms against oxidative stress [3]. The expression of endogenous OXR1 protein is induced under oxidative stress conditions in human cells $[4,5]$. Previous studies suggested that OXR1 inhibits the generation of oxidative DNA damage by inhibiting oxidative stress to protect cellular survival and genomic integrity [6-11]. DNA damage response (DDR) induces cell cycle checkpoints and DNA damage repair system $[1,12]$. Cell cycle checkpoints inhibit cell cycle progression and provide ample

(c) The Author(s). 2020 Open Access This article is licensed under a Creative Commons Attribution 4.0 International License, which permits use, sharing, adaptation, distribution and reproduction in any medium or format, as long as you give appropriate credit to the original author(s) and the source, provide a link to the Creative Commons licence, and indicate if changes were made. The images or other third party material in this article are included in the article's Creative Commons licence, unless indicated otherwise in a credit line to the material. If material is not included in the article's Creative Commons licence and your intended use is not permitted by statutory regulation or exceeds the permitted use, you will need to obtain permission directly from the copyright holder. To view a copy of this licence, visit http://creativecommons.org/licenses/by/4.0/ The Creative Commons Public Domain Dedication waiver (http://creativecommons.org/publicdomain/zero/1.0/) applies to the data made available in this article, unless otherwise stated in a credit line to the data. 
time for DNA repair. When DNA damage is sufficiently repaired, the cell cycle continues to progress. If the state of DNA damage exceeds repair capacity, cell death is provoked. Thus, DDR allows genomic stable cells to survive and prevents the proliferation of genomically unstable cells $[1,13,14]$. OXR1 is thought to affect the activation of G2-phase cell cycle checkpoint through oxidative stress inhibition [11].

Recently, we demonstrated that human OXR1 protein participates in genomic stability through the reduction of oxidative stress and in cell cycle checkpoint in cells irradiated with gamma-rays ( $\gamma$-rays) [15]. In addition, the function of OXR1 in the regulation of G2-phase arrest was partially independent of oxidative stress. This phenomenon may be due to DNA strand breaks generated directly by depositing energy on the DNA strand and in addition to the indirect damage caused by ROS [16]. These results implied that OXR1 functions in the response to ROS-independent DNA damage.

DNA double-strand breaks (DSBs) are generated by both ROS-dependent and ROS-independent manner $[1,16]$. Methyl methanesulfonate (MMS), one of DNA-alkylating reagents, methylates DNA bases, and the methylated sites are converted to AP sites and strand breaks in the process of DNA damage repair $[1,17]$. Irradiation with heavy-ion beams, such as carbon- and iron-ion beams, at high linear energy transfer (LET) has biological effects mainly through ROSindependent mechanism, which is different from low LET irradiation, including $\gamma$-rays. Therefore, DNA damage generated by irradiation with heavy-ion beams are DSBs and more complex DNA lesions than low LET radiation [18-21]. Thus, irradiation with heavyion beams and treatment with MMS can generate DNA damage in a ROS-independent manner.

In the present study, to clarify the functions of OXR1 in response to ROS-independent DNA damage, we treated HeLa cells and OXR1-depleted HeLa cells with
MMS and heavy-ion beams. Our study suggested that OXR1 plays an important role in the response to DNA damage.

\section{Materials and methods}

Cells and treatment

OXR1-depleted HeLa cells were established as described previously [15]. Cells were cultured in Dulbecco's modified Eagle's medium (low glucose, Wako Pure Chemical Industries) supplemented with $10 \%$ fetal bovine serum at $37^{\circ} \mathrm{C}$ in a humidified incubator supplied with $5 \% \quad \mathrm{CO}_{2}$. Methyl methanesulfonate (MMS, Tokyo Chemical Industry) was dissolved in distilled water at the concentration of $1 \mathrm{M}$. Irradiation by heavy-ion beams was performed in the Heavy Ion Medical Accelerator in Chiba (HIMAC) at the $\mathrm{Na}$ tional Institute of Radiological Sciences (NIRS) in Japan. Details of the treatment conditions are described in the results or figure legends.

\section{Colony formation assay}

For MMS treatment, 100 cells were seeded in $60-\mathrm{mm}$ diameter dishes and incubated for 5-6h. The cells were constitutively treated with 80,160 or $320 \mu \mathrm{M}$ MMS. Plating efficiency was more than $90 \%$ in OXR1-depleted cells and control cells. For irradiation with heavy-ion beams, 500 or 1000 cells were seeded in T25 culture flasks (Falcon 3014 or CORNING 25 $\mathrm{cm}^{2}$ Triangular Angled Neck Cell Culture Flask) since the plating efficiency was around $30-50 \%$, and incubated for $10-16 \mathrm{~h}$. The cells were irradiated with carbon-ion beams $(0,2,4$ or $6 \mathrm{~Gy}, 290 \mathrm{MeV} /$ nucleon, $87.0 \mathrm{keV} / \mu \mathrm{m})$ or iron-ion beams $(0,1,3$ or $5 \mathrm{~Gy}, 500$ $\mathrm{MeV} /$ nucleon, $200 \mathrm{keV} / \mu \mathrm{m})$. After 10-14 days, the samples were stained with crystal violet dissolved in $20 \%$ methanol. The number of colonies containing more than 50 cells was counted. a

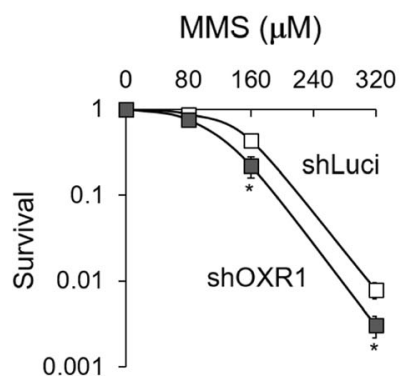

b

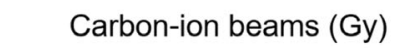

c

\section{Iron-ion beams (Gy)}

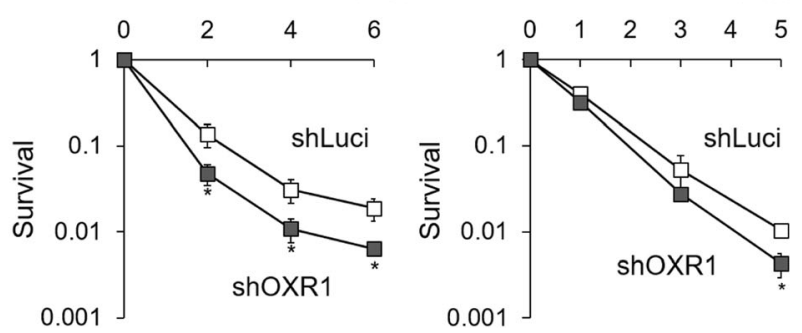

Fig. 1 Sensitivity to MMS and heavy-ion beams. a-c Quantification of cellular survival. OXR1-depleted cells (shOXR1; closed square) and control cells (shLuci; open square) were (a) exposed constitutively to MMS (80, 160 or $320 \mu \mathrm{M})$ for 11 days, or irradiated with (b) carbon-ion beams $(0,2,4$ or $6 \mathrm{~Gy}, 290 \mathrm{MeV} / \mathrm{nucleon}, 87.0 \mathrm{keV} / \mu \mathrm{m}$ ) or (c) iron-ion beams (0, 1, 3 or $5 \mathrm{~Gy}, 500 \mathrm{MeV} /$ nucleon, $200 \mathrm{keV} / \mu \mathrm{m})$. Cellular survival was analyzed by the colony formation assay. Means \pm s.d. of $n=$ three independent experiments, ${ }^{*} p<0.05$, two-tailed Student's t-test 


\section{Cell synchronization}

Cell synchronization to G1/S-phase was performed as described previously [15]. Cells were seeded in culture dishes and cultured for one or two days. The cells were treated with $2.5 \mathrm{mM}$ thymidine (Wako Pure Chemical Industries) for $20-24 \mathrm{~h}$, followed by incubation in fresh medium for $10 \mathrm{~h}$. The cells were treated with $1 \mathrm{mM}$ hydroxyurea (ACROS ORGANICS). After $14-16 \mathrm{~h}$, the a
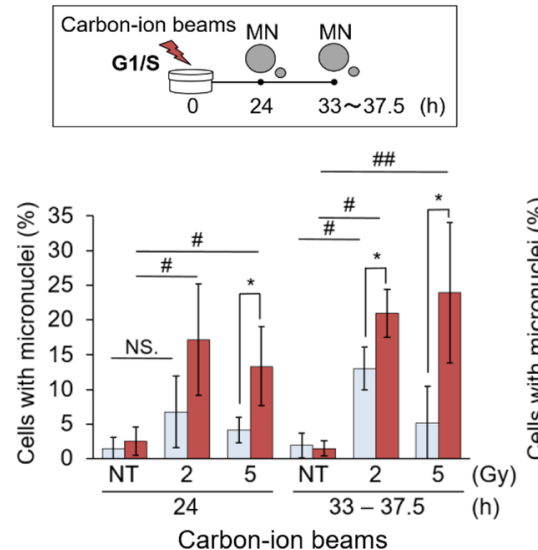

$\square$ shLuci b
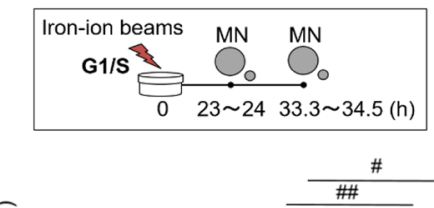

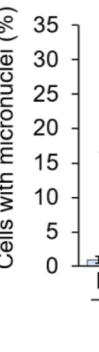

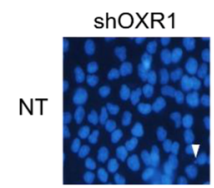

d
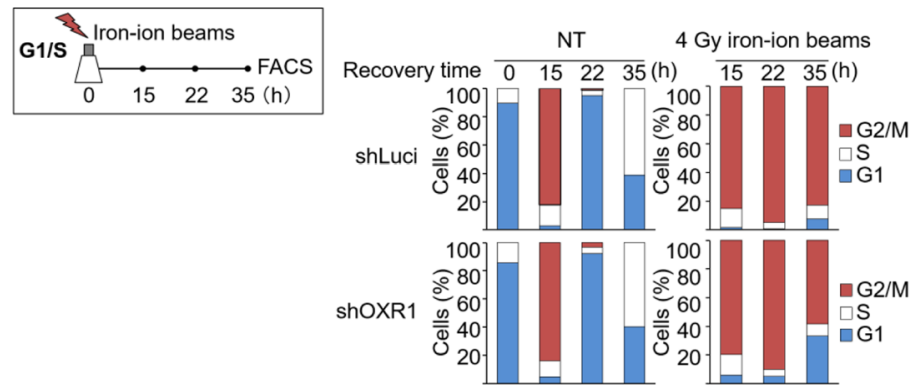

e
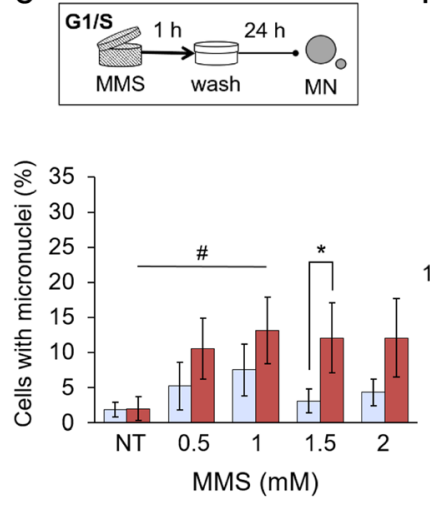

f

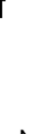

NT

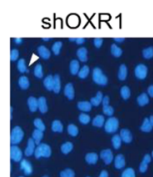

$1.5 \mathrm{mM}$ MMS

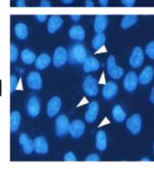

$24 \mathrm{hrs}$

g

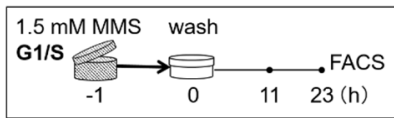

$1.5 \mathrm{mM}$ NT MMS $1 \mathrm{~h}$ Recovery time $0 \overline{1123}(\mathrm{~h})$

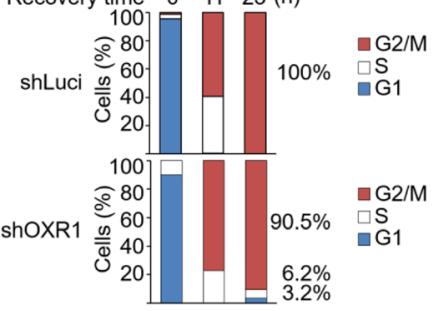

$\square$ shLuci $\square$ shOXR1

Fig. 2 MN formation level after treatment with MMS and heavy-ion beams. a-c Quantification of the MN formation level. OXR1-depleted HeLa cells or control cells were synchronized at G1/S-phase. The cells were irradiated with (a) carbon-ion beams (2 or 5 Gy, 290 MeV/nucleon, 83.8-86 $\mathrm{keV} / \mu \mathrm{M}$ ) or (b) iron-ion beams (2 or $4 \mathrm{~Gy}, 500 \mathrm{MeV} /$ nucleon, $200 \mathrm{keV} / \mu \mathrm{m})$. c Representative fluorescent images of DAPI-stained nuclei. Arrowheads indicate micronuclei. $\mathbf{d}$ Distribution of the cell cycle. Cells were synchronized at G1/S phase. The cells were irradiated with 4 Gy of iron-ion beams (500 MeV/nucleon, $200 \mathrm{keV} / \mu \mathrm{m}$ ). After the indicated times, cell cycle distributions were measured by FACS. NT: no treatment. e Quantification of the MN formation level in cells treated with MMS $(0.5,1,1.5$ or $2 \mathrm{mM})$ for $1 \mathrm{~h}$. $\mathbf{f}$ Representative fluorescent images of DAPI-stained nuclei. $\mathbf{g}$ Distribution of the cell cycle in cells treated with $1.5 \mathrm{mM} \mathrm{MMS}$ for $1 \mathrm{~h}$. The percentage of cells with micronuclei was calculated ( $a, b \geq 1000, d$. $\geq$ 460 per condition per experiment). Means \pm s.e.m. of $n=$ three independent experiments, $\# p<0.05, \# \# p<0.01$, three-way ANOVA with Dunnett's multiple comparisons test, ${ }^{*} p<0.05$, two-tailed Welch's and Student's t-test. NT, no treatment 
cells were synchronized to G1/S phase, and then exposed to DNA-damaging agents.

\section{Quantification of micronucleus (MN) formation}

We used MN formation as biological endpoint for genotoxic effects and chromosomal instability [22]. In 35-mm diameter dishes, $0.7-0.8 \times 10^{5}$ cells/dish were seeded and synchronized at G1/S-phase. The cells were treated with heavy-ion beams or MMS as described in the figure legends. After recovery incubation, the cells were fixed with PBS / 4\% paraformaldehyde for $15 \mathrm{~min}$ at $4{ }^{\circ} \mathrm{C}$ and permeabilized with $0.1 \%$ Triton $\mathrm{X}-100$ for $5 \mathrm{~min}$ at room temperature. Nuclei were stained with $5 \mu \mathrm{g} / \mathrm{ml}$ of DAPI for $5 \mathrm{~min}$ followed by washing with PBS. Micronuclei were observed using the fluorescence microscope OLYMPUS IX70 equipped with OLYMPUS DP50 using the $10 \times$ or $20 \times$ objective lens. The percentage of cells with micronuclei ( $\geq 460$ cells per condition per experiment) was calculated.

\section{Cell cycle analysis}

Cells were seeded in T25 flasks (Falcon) for irradiation or 60-mm diameter dishes for MMS treatment and cultured to approximately $20-40 \%$ confluency for one or two days. The cells were synchronized in G1/S-phase, followed by irradiation or MMS treatment. The cells were fixed with $70 \%$ ethanol at $-20^{\circ} \mathrm{C}$ for more than 16 $\mathrm{h}$. The cells were counter-stained with PBS/ $50 \mu \mathrm{g} / \mathrm{ml}$ of propidium iodide (PI, Sigma-Aldrich) containing $5 \mu \mathrm{g} / \mathrm{ml}$ of RNase. Fluorescence signal was detected by FACS Calibur (BD Biosciences) for 20,000 cells per sample and cell cycle distribution was analyzed using the BD CellQuest Pro Software (BD Biosciences).

\section{Western blot}

Western blot was performed as described previously [15]. Briefly, whole cell lysates were separated by SDSPAGE, followed by transfer to nitrocellulose membranes. The membranes were blocked with skim milk, and then proteins in the membranes were reacted with antibodies. Signals were developed using chemiluminescence (ECL, Amersham) and the membranes were then exposed to X-ray film (FUJIFILM). Images were analyzed by ImageJ 1.5a (Wayne Rasband National Institute of Health, USA http://imagej.nih.gov/ij). Antibodies used were: OXR1 (purified previously [15], 1:3000), Beta-actin (A5361, Sigma-Aldrich, 1:10,000), Beta-Tubulin (sc-9104, Santa Cruz Biotechnology, 1:1000), Rabbit-IgG-HRP (sc-2030, Santa Cruz Biotechnology, 1:5000) and Mouse-IgG-HRP (sc-2005, Santa Cruz Biotechnology, 1:5000).

\section{Statistical analysis}

The data for the statistical assay were from more than three independent experiments. The data are presented as the mean \pm s.e.m. or s.d.. Statistical differences between conditions were analyzed by ANOVA with the Welch's, Student's t-test or the Dunnett's test using $\mathrm{R}$ 3.5.0 GUI 1.70 El Capitan build (7521), S. Urbanek, H.-J. Bibiko, \& Stefano M. Iacus, See http://www.R-project. org for more information. $p$-values $<0.05$ were considered significant.

\section{Results and discussion \\ Sensitivity to MMS and heavy-ion beams}

To investigate whether OXR1 defends against ROSindependent DNA damage, cells were treated with MMS or irradiated with heavy-ion beams and the cell viability was evaluated by colony formation assay. OXR1depleted cells exhibited significantly higher sensitivity to MMS treatment, carbon-ion beams and iron-ion beams irradiation than control cells (Fig. 1a-c). These results suggested that OXR1 plays an important role in defends against ROS-independent DNA damage, which is mainly consists of DNA strand breaks.

The previous reports and our recent study demonstrated that depletion of OXR1 exhibited increased sensitivity to oxidative stress, indicating that OXR1 functions in protection of cells from genotoxic oxidative stress $[5,9,15]$. Together with present study, OXR1 maintains cellular survival in the response to DNA damage, and the source of DNA damage activating OXR1 is not limited to ROS.

\section{MN formation and cell cycle distribution}

Recently our study demonstrated that OXR1 depletion increases $\mathrm{MN}$ formation and accumulation of cells in G2/M-phase after treatment with hydrogen peroxide or irradiation with $\gamma$-rays to the synchronized cells in G1/ S-phase [15]. We found that the increased level of MN formation in OXR1-depleted cells were caused by both ROS-dependent and -independent factors [15]. However, the generation of $\mathrm{MN}$ through ROS-independent mechanism needs further verification. To investigate whether OXR1 depletion also induces MN formation actually by ROS-independent DNA damage, the synchronized cells in G1/S-phase were irradiated with heavy-ion beams, and the number of cells containing MN was measured.

Cells were irradiated with heavy-ion beams at the dose in which $1 \%$ of survival rate was detected following our previous experiment [15]. We observed MN formation and cell cycle distribution until $24 \mathrm{~h}$ after irradiation with $\gamma$-rays in previous report [15]. Since it has been reported that the duration of G2-phase arrest in the cells irradiated with heavy-ion beams is longer than low LET radiation [23, 24], we decided to observe $\mathrm{MN}$ formation in irradiated cells over a period of time longer than $24 \mathrm{~h}$ after irradiation. 
At $24 \mathrm{~h}$ or $33-37.5 \mathrm{~h}$ after irradiation with $5 \mathrm{~Gy}$ of carbon-ion beams, MN formation level in OXR1depleted cells was significantly increased compared with that in control cells (Fig. 2a). Similarly, at 33.3-34.5 h after irradiation with 4 Gy of iron-ion beams, the level of MN formation in OXR1-depleted cells was higher than that in control cells (Fig. 2b, c). In cells in which MN formation is accelerated, more DNA lesions are unrepaired [22, 25]. We next analyzed the cell cycle distribution of the cells synchronized G1/S-phase, followed by irradiation with iron-ion beams. At $35 \mathrm{~h}$ after irradiation with 4Gy of iron-ion beams, a smaller fraction of OXR1-depleted cells in G2/M-phase was observed compared with control cells (Fig. 2d). In the case of MMS treatment, higher $\mathrm{MN}$ formation level and a smaller fraction of G2/M-phase were observed in OXR1depleted cells (Fig. 2e-g), as similar to iron-ion beam treatment.

Less distribution of cells in G2/M-phase compared with control and untreated cells most likely indicates partial defects of G2-phase checkpoint control, which is one of DNA damage checkpoint control [26]. If the G2-phase checkpoint control is insufficient, ROSindependent DNA strand breaks generated by heavyion beams and MMS might not be fully repaired, leading to genetic aberration and cell death. Taken together, our results suggest that OXR1 participates in genomic stability through regulation of the G2phase checkpoint.

The main mechanism by which MMS treatment damages DNA is alkylation, not oxidation by $\operatorname{ROS}[17,27]$. In cells irradiated with heavy-ion beams, the effects of ROS-independent factors on cellular components, including DNA, have been thought to be larger than those of ROS [21, 28-31]. Thus, MMS treatment and heavyion beam irradiation provoke mainly ROS-independent damage. Furthermore, we previously showed that the depletion of OXR1 suppresses the G2-phase checkpoint, ROS-independently [15]. Together with the previous studies, the present study shows that OXR1 functions in the response to DNA damage generated in a ROSindependent manner.

\section{Protein level of OXR1}

To investigate whether the protein level of OXR1 is increased by ROS-independent genotoxic stresses, HeLa cells were treated with $1.5 \mathrm{mM}$ MMS for $1 \mathrm{~h}$ or $2.5 \mathrm{~Gy}$ of irradiated with carbon-ion beams, and the protein level of endogenous OXR1 was analyzed by Western blot. The experiment was repeated twice and a representative blot is shown in Fig. 3. The OXR1 protein level increased to approximately two-fold just after MMS treatment and decreased to a steady-state level $4 \mathrm{~h}$ after treatment (Fig. 3a). When HeLa cells were irradiated with carbon-ion beams, OXR1 protein level reached a maximum by $8 \mathrm{~h}$ after irradiation, and this higher protein level continued until at least $72 \mathrm{~h}$ after irradiation (Fig. 3b).

Compared with MMS-treatment, delayed elevation of OXR1 protein level was observed in carbon-ion beams irradiation. This might be due to that irradiation of heavy-ion beams produce more complex DNA damage than MMS-treatment. Thus, the expression of OXR1 protein was induced by both MMS and carbon-ion beams. One of possibility is that increased amount of OXR1 protein might require for maintenance of G2-phase checkpoint control. So far, it was known that the protein level of OXR1 is induced by the oxidative stress $[4,5]$. This study revealed that the OXR1 protein level is also increased by treatment with MMS or heavy-ion beams, suggesting that OXR1 protein is involved in the regulation of DDR (Fig. 2), even when the DNA damage is not triggered by oxidative stress. a

HeLa WT

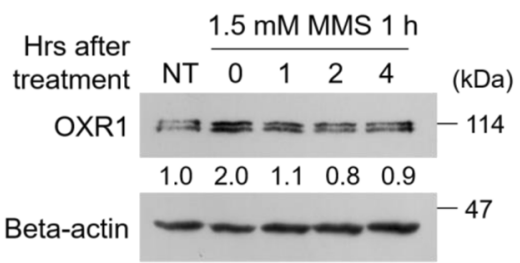

b

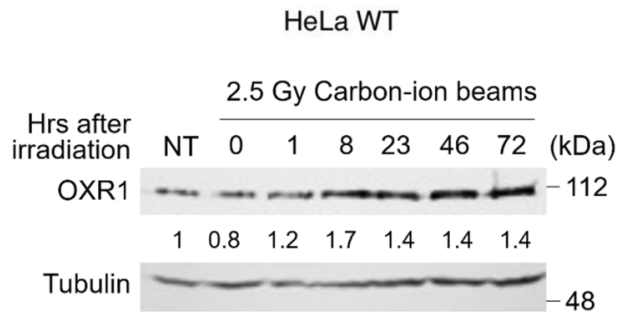

Fig. 3 Induction of OXR1 protein level. a, b OXR1 protein level. HeLa cells (indicated as HeLa WT) were treated with (a) 1.5 mM MMS for 1 h or (b) $2.5 \mathrm{~Gy}$ of carbon-ion beams, and the cells were incubated for the indicated times. The OXR1 protein level in crude extracts was assessed by Western blot using anti-OXR1 antibody. Proteins were separated with 10\% SDS-PAGE gel. OXR1 protein was detected as (a) two or (b) single bands because of the difference in each gel running time. It has been confirmed that both two bands are OXR1 in our previous study [15]. Relative OXR1 protein levels (vs. no treatment) normalized by beta-actin or tubulin are shown below OXR1 blots. The size of marker proteins is shown on the right of the image. Images were analyzed by Image J software. NT, no treatment 


\section{Conclusions}

In this study, we found that the OXR1 depletion increased cell sensitivity and the OXR1 protein level is increased in response to ROS-independent DNA damage. OXR1 protein helps maintain genome stability directly against ROS-dependent and independent DNA damage.

\section{Abbreviations}

ROS: Reactive oxygen species; DDR: DNA damage response; OXR1: Oxidation resistance 1; MMS: Methyl methanesulfonate; MN: Micronucleus; LET: Linear energy transfer; DSB: Double strand-break

\section{Acknowledgements}

We thank Mr. Yukihiro Yoshikawa for helping conduct experiments with heavy-ion beams and Dr. Junya Kobayashi for helping analyze cell cycle. We also thank Dr. Elena Zaharieva for critical reading and editing of this manuscript.

\section{Authors' contributions}

A.M. and Q.Z.A. designed the project. A.M. designed the experiments. A.M., K.H. and M.S. performed the experiments. A.M. analyzed the data. A.M., K.H. and Q.Z.A. wrote the manuscript. All authors commented on and approved the manuscript.

\section{Funding}

Irradiation experiments were performed at the Joint Usage/Research Center at the Radiation Biology Center, Kyoto University. This study was supported by the Research Project with Heavy lons at National Institute of Radiological Sciences (NIRS)-Medical Accelerator in Chiba (HIMAC) [12 J-393 to Q.Z.A]. This study was supported in part by Grants-in-Aid for Scientific Research from the Ministry of Education, Culture, Sports, Science and Technology of Japan [19 K12320 to Q.Z.A].

\section{Availability of data and materials}

All data generated or analyzed during this study are included in this published article.

\section{Ethics approval and consent to participate}

Not applicable.

\section{Consent for publication}

Not applicable.

\section{Competing interests}

The authors declare that they have no competing interests.

\section{Author details}

${ }^{1}$ Laboratory of Stress Response Biology, Department of Zoology, Division of Biological Sciences, Graduate School of Science, Kyoto University, Kitashirakawa Oiwake-cho, Sakyo-ku, Kyoto 606-8502, Japan. ²Department of Experimental Oncology, Research Institute for Radiation Biology and Medicine, Hiroshima University, 1-2-3 Kasumi, Minami-ku, Hiroshima 7348553, Japan. ${ }^{3}$ Department of Biochemistry, Fukuoka Dental College, 2-15-1 Tamura, Sawara-ku, Fukuoka 814-0193, Japan. ${ }^{4}$ Department of Basic Medical Sciences for Radiation Damages, National Institute of Radiological Sciences, National Institutes for Quantum and Radiological Science and Technology, 4-9-1 Anagawa, Inage-ku, Chiba 263-8555, Japan.
\end{abstract}

Received: 6 August 2020 Accepted: 29 October 2020

Published online: 10 November 2020

\section{References}

1. Ciccia A, Elledge SJ. The DNA damage response: making it safe to play with knives. Mol Cell. 2010;40:179-204.

2. Sies H, Berndt C, Jones DP. Oxidative stress. Annu Rev Biochem. 2017;86: 715-48

3. Finelli MJ, Oliver PL. TLDc proteins: new players in the oxidative stress response and neurological disease. Mamm Genome. 2017;28:395-406.

4. Elliott NA, Volkert MR. Stress induction and mitochondrial localization of Oxr1 proteins in yeast and humans. Mol Cell Biol. 2004;24:3180-7.
5. Zhang $X$, Zhang S, Liu $X$, et al. Oxidation resistance 1 is a novel senolytic target. Aging Cell. 2018:e12780. https://doi.org/10.1111/acel.12780.

6. Volkert MR, Elliott NA, Housman DE. Functional genomics reveals a family of eukaryotic oxidation protection genes. Proc Natl Acad Sci U S A. 2000;97:14530-5.

7. Oliver PL, Finelli MJ, Edwards B, et al. Oxr1 is essential for protection against oxidative stress-induced neurodegeneration. PLoS Genet. 2011;7. https://doi. org/10.1371/journal.pgen.1002338.

8. Murphy KC, Volkert MR. Structural/functional analysis of the human OXR1 protein: identification of exon 8 as the anti-oxidant encoding function. BMC Mol Biol. 2012;13:26.

9. Yang M, Luna L, Sørbø JG, et al. Human OXR1 maintains mitochondrial DNA integrity and counteracts hydrogen peroxide-induced oxidative stress by regulating antioxidant pathways involving p21. Free Radic Biol Med. 2014;77:41-8.

10. Sanada $Y$, Asai S, Ikemoto $A$, et al. Oxidation resistance 1 is essential for protection against oxidative stress and participates in the regulation of aging in Caenorhabditis elegans. Free Radic Res. 2014;48:919-28.

11. Yang M, Lin X, Rowe A, et al. Transcriptome analysis of human OXR1 depleted cells reveals its role in regulating the p53 signaling pathway. Sci Rep. 2015;5:1-12.

12. Lord CJ, Ashworth A. The DNA damage response and cancer therapy. Nature. 2012:481:287-94.

13. Shaltiel IA, Krenning $L$, Bruinsma W, et al. The same, only different - DNA damage checkpoints and their reversal throughout the cell cycle. J Cell Sci. 2015;128:607-20.

14. Otto T, Sicinski P. Cell cycle proteins as promising targets in cancer therapy. Nat Rev Cancer. 2017;17:93-115.

15. Matsui A, Kobayashi J, Kanno S-I, et al. Oxidation resistance 1 prevents genome instability through maintenance of G2/M arrest in gamma-rayirradiated cells. J Radiat Res. 2020:61:1-13.

16. Powell S, McMillan TJ. DNA damage and repair following treatment with ionizing radiation. Radiother Oncol. 1990;19:95-108.

17. Fu D, Calvo JA, Samson LD. Balancing repair and tolerance of DNA damage caused by alkylating agents. Nat Rev Cancer. 2012;12:104-20.

18. Hada M, Georgakilas AG. Formation of clustered DNA damage after highLET irradiation : a review. J Radiat Res. 2008;49:203-10.

19. Sage E, Shikazono N. Radiation-induced clustered DNA lesions: repair and mutagenesis. Free Radic Biol Med. 2016. https://doi.org/10.1016/j. freeradbiomed.2016.12.008

20. Hagiwara Y, Oike T, Niimi A, et al. Clustered DNA double-strand break formation and the repair pathway following heavy-ion irradiation. J Radiat Res. 2018;60:69-79.

21. Dubrovska A, Mechthild K, Baumann M. The biology of cancer treatment: biological effect of radiotherapy on cancer cells. In: Pezzella F, Tavassoli M, Kerr DJ, editors. Oxford Textbook of Cancer Biology. Oxford: Oxford University Press; 2019. https:/doi.org/10.1093/med/9780198779452.003.0030.

22. Fenech M, Kirsch-Volders M, Natarajan AT, et al. Molecular mechanisms of micronucleus, nucleoplasmic bridge and nuclear bud formation in mammalian and human cells. Mutagenesis. 2011;26:125-32.

23. Asaithamby A, Hu B, Chen DJ. Unrepaired clustered DNA lesions induce chromosome breakage in human cells. Proc Natl Acad Sci U S A. 2011;108:8293-8.

24. Nakajima NI, Brunton $H$, Watanabe $R$, et al. Visualisation of $\mathrm{YH} 2 \mathrm{AX}$ foci caused by heavy ion particle traversal; distinction between core track versus non-track damage. PLoS One. 2013;8. https://doi.org/10.1371/journal.pone.0070107.

25. Crasta K, Ganem NJ, Dagher R, et al. DNA breaks and chromosome pulverization from errors in mitosis. Nature. 2012;482:53-8.

26. Liu Q, Guntuku S, Cui XS, et al. Chk1 is an essential kinase that is regulated by ATR and required for the G2/M DNA damage checkpoint. Genes Dev. 2000;14:1448-59.

27. Degtyareva NP, Saini N, Sterling JF, et al. Mutational signatures of redox stress in yeast single-strand DNA and of aging in human mitochondrial DNA share a common feature. PLoS Biol. 2019;17:e3000263.

28. Ito $\mathrm{A}$, Nakano $\mathrm{H}$, Kusano $\mathrm{Y}$, et al. Contribution of indirect action to radiationinduced mammalian cell inactivation: dependence on photon energy and heavy-ion LET. Radiat Res. 2006:165:703-12.

29. Hirayama R, Ito A, Tomita M, et al. Contributions of direct and indirect actions in cell killing by high-LET radiations. Radiat Res. 2009;171:212-8.

30. Yoshikawa Y, Yamasaki A, Takatori $K$, et al. Excess processing of oxidative damaged bases causes hypersensitivity to oxidative stress and low dose rate irradiation. Free Radic Res. 2015:49:1239-48.

31. Tokuyama $Y$, Furusawa $Y$, Ide $H$, et al. Role of isolated and clustered DNA damage and the post-irradiating repair process in the effects of heavy ion beam irradiation. J Radiat Res. 2015;56:446-55.

\section{Publisher's Note}

Springer Nature remains neutral with regard to jurisdictional claims in published maps and institutional affiliations. 